preliminary results. We propose proactive vaccination of Ethiopian wolves across their distribution as an effective and urgently needed strategy to protect the species from extinction. This program should be part of an integrated disease control plan that also includes controlling disease in domestic dogs, limiting contact between dogs and wolves, and conducting policy and education interventions to reduce the size and roaming behavior of local dog populations (2).

\section{Acknowledgments}

We thank the Ethiopian Wildlife Conservation Authority and Environment and Forest and Wildlife Protection and Development Authority (Amhara National Regional State) for support and permission to work in Delanta. We thank Delanta and Gubalaftu Waredas and Wolf Ambassadors, and Leigh Thorne and Daisy Jennings for excellent technical assistance.

The work was funded by the Born Free Foundation and the Wildlife Conservation Network. The work undertaken by Animal and Plant Health Agency is funded by a grant (SEV3500) from the UK Department for Environment, Food and Rural Affairs, Scottish and Welsh Governments.

The animal care and use protocols for the ethical handling of Ethiopian wolves were approved by the Ethiopian Wildlife Conservation Authority and the University of Oxford's Local Ethical Review Process (Zoology ERC; case no. ZERC040905) and adhere to the United Kingdom's ASPA regulations (1986).

Dr. Marino is a conservation ecologist with the University of Oxford's Wildlife Conservation Research Unit (WildCRU), Oxford, UK, and is science director of the Ethiopian Wolf Conservation Programme. She is interested in the ecology and conservation of threatened carnivores, and coordinates Ethiopian Wolf Conservation Programme monitoring efforts, looking at the demography of the rare and endangered Ethiopian wolf, interactions with free-ranging dogs, and the impact of viral diseases.

\section{References}

1. Mähl P, Cliquet F, Guiot A-L, Niin E, Fournials E, Saint-Jean N, et al. Twenty year experience of the oral rabies vaccine SAG2 in wildlife: a global review. Vet Res (Faisalabad). 2014;45:77. http://dx.doi.org/10.1186/s13567-014-0077-8

2. Strategic planning for Ethiopian wolf conservation. Oxford (UK): International Union for the Conservation of Nature, Canid Specialist Group; 2011. p. 79 [cited 2017 May 25]. https://portals.iucn.org/library/efiles/documents/2011-090.pdf

3. Gordon CH, Banyard AC, Hussein A, Laurenson MK, Malcolm JR, Marino J, et al. Canine distemper in endangered Ethiopian wolves. Emerg Infect Dis. 2015;21:824-32. http://dx.doi.org/10.3201/ eid2105.141920

4. Haydon DT, Randall DA, Matthews L, Knobel DL, Tallents LA, Gravenor MB, et al. Low-coverage vaccination strategies for the conservation of endangered species. Nature. 2006;443:692-5. http://dx.doi.org/10.1038/nature05177

5. Haydon DT, Laurenson MK, Sillero-Zubiri C. Integrating epidemiology into population viability analysis: managing the risk

posed by rabies and canine distemper to the Ethiopian wolf. Conserv Biol. 2002;16:1372-85. http://dx.doi.org/10.1046/j. 1523-1739.2002.00559.x

6. Johnson N, Mansfield KL, Marston DA, Wilson C, Goddard T, Selden D, et al. A new outbreak of rabies in rare Ethiopian wolves (Canis simensis). Arch Virol. 2010;155:1175-7. http://dx.doi.org/ 10.1007/s00705-010-0689-x

7. Sillero-Zubiri C. Field immobilization of Ethiopian wolves (Canis simensis). J Wildl Dis. 1996;32:147-51. http://dx.doi.org/ 10.7589/0090-3558-32.1.147

8. Laurenson K, Sillero-Zubiri C, Thompson H, Shiferaw F, Thirgood S, Malcolm J. Disease as a threat to endangered species: Ethiopian wolves, domestic dogs and canine pathogens. Anim Conserv. 1998;1:273-80. http://dx.doi.org/10.1111/j.1469-1795. 1998.tb00038.x

9. Marino J, Sillero-Zubiri C, Gottelli D, Johnson PJ, Macdonald DW. The fall and rise of Ethiopian wolves: lessons for conservation of long-lived, social predators. Anim Conserv. 2013;16:621-32. http://dx.doi.org/10.1111/acv.12036

10. Sillero-Zubiri C, Marino J, Gordon CH, Bedin E, Hussein A, Regassa F, et al. Feasibility and efficacy of oral rabies vaccine SAG2 in endangered Ethiopian wolves. Vaccine. 2016;34:4792-8. 10.1016/j.vaccine.2016.08.021 http://dx.doi.org/10.1016/ j.vaccine.2016.08.021

Address for correspondence: Jorgelina Marino, Wildlife Conservation Research Unit, University of Oxford, The Recanati-Kaplan Centre, Tubney House, Tubney, Oxford OX13 5QL, UK; email: jorgelina.marino@zoo.ox.ac.uk

\title{
High Abundance and Genetic Variability of Atypical Porcine Pestivirus in Pigs from Europe and Asia
}

\section{Alexander Postel, Denise Meyer, Gökce Nur Cagatay, Francesco Feliziani, Gian Mario De Mia, Nicole Fischer, Adam Grundhoff, Vesna Milićević, Ming-Chung Deng, Chia-Yi Chang, Hua-Ji Qiu, Yuan Sun, Michael Wendt, Paul Becher}

Author affiliations: University of Veterinary Medicine, Hannover, Germany (A. Postel, D. Meyer, G.N. Cagatay, M. Wendt, P. Becher); Istituto Zooprofilattico Sperimentale dell'Umbria e delle Marche, Perugia, Italy (F. Feliziani, G.M. De Mia); University Medical Center Hamburg-Eppendorf, Hamburg, Germany (N. Fischer); Heinrich Pette Institute, Hamburg (A. Grundhoff); Institute of Veterinary Medicine of Serbia, Belgrade, Republic of Serbia (V. Milićević); Animal Health Research Institute, 
New Taipei City, Taiwan (M.-C. Deng, C-Y. Chang); Harbin Veterinary Research Institute, Harbin, China (H.-J. Qiu, Y. Sun)

DOI: https://doi.org/10.3201/eid2312.170951

Atypical porcine pestivirus (APPV) was recently reported to be associated with neurologic disorders in newborn piglets. Investigations of 1,460 serum samples of apparently healthy pigs from different parts of Europe and Asia demonstrate a geographically wide distribution of genetically highly variable APPV and high APPV genome and antibody detection rates.

$\mathrm{P}$ estiviruses are highly variable RNA viruses within the family Flaviviridae. The recently discovered atypical porcine pestivirus (APPV) is capable of inducing neurologic disorder in its host, like other pathogens of this family (e.g., tick-borne encephalitis virus, Zika virus). Several recently published reports demonstrate that APPV is a prominent cause of virus-induced congenital tremor in pigs (1-4). Serum samples from healthy but viremic animals can induce birth of clinically affected offspring when experimentally transferred to sows during gestation $(1,2)$. So far, APPV presence has been reported from the United States, some countries within Europe, and China $(2,4-7)$. The economic relevance of APPV-related losses in pig production remains to be determined, but estimation revealed a drop in reproductive performance of $10 \%$ in an affected farm (4). Early data from the United States and Germany suggested a relatively high abundance $(2.4 \%-$ $22 \%)$ of APPV genomes in apparently healthy pigs $(3,6,8)$ that likely play an important epidemiologic role as virus carriers. We investigated APPV genome and antibody abundance in healthy pigs from different parts of Europe and Asia to provide insight into genetic diversity of this novel pathogen.

We tested 1,460 serum samples from Germany, Great Britain, Italy, Serbia, Switzerland, mainland China, and Taiwan by using an APPV-specific PCR and an indirect APPV ELISA, as previously described $(3,9)$. The sample set comprised 369 serum samples from Germany that were previously screened for the presence of APPV genomes (3). For our study, serum samples were taken from apparently healthy pigs within the framework of national veterinary health management in concordance with national legal and ethical regulations.

For APPV genome detection, we conducted a PCR targeting the nonstructural protein (NS) 3 encoding region and confirmed specificity of amplification by gel electrophoresis (3). We detected APPV genomes in domestic pigs from all investigated regions. In total, 130 (8.9\%) of the 1,460 tested samples were APPV genome positive (Figure). Genome detection rates ranged from 2.3\% (2/86 samples from Great Britain) to $17.5 \%$ (35/200 samples from Italy). Moreover, we demonstrated that APPV was abundant in Asia; we detected the APPV genome in $11 / 219$ samples (5\%) from mainland China and 22/200 samples (11\%) from Taiwan.

We used individual samples with high genome loads to generate amplicons in a seminested PCR and subsequently performed Sanger sequencing (FlexiRun, LGC Genomics, Berlin, Germany). We generated 20 different APPV NS3 sequences from apparently healthy pigs of all countries (sequences deposited into GenBank under accession nos. MF279213-32). Genetic differences reflect geographic origin to a low degree (Figure); genetic variability even within a country is remarkably high (e.g., Germany and Italy). Genetic analyses including sequence data obtained from samples of diseased piglets revealed no correlation of pathogenicity with certain genetic variants (Figure).

In addition to the NS3 fragments, we determined APPV complete coding sequences (CDS) from 1 sample from a healthy pig from China (deposited into GenBank under accession no. MF167292) and 2 samples (accession nos. MF167290 and MF167291) obtained from pigs during outbreaks of congenital tremors in Germany $(3,9)$. We performed next-generation sequencing as previously described (3). The outbreak isolates from Germany were almost identical $(0.2 \%$ genetic distance) and were similar to an isolate from northern Germany (accession no. LT594521). The APPV from China had a unique 93-nt deletion in the NS5A encoding region. A similar genome (97.9\% identity) is lacking this deletion (Guangxi Province; accession no. KY652092). The biological relevance of the deletion remains elusive, but classical pestiviruses show a remarkable genetic tolerance in this genomic region (10). The sequence data we obtained reveal a high genetic variability (up to $21 \%$ genetic distance), which is comparable to that of classical swine fever virus (online Technical Appendix, https:// wwwnc.cdc.gov/EID/article/23/12/17-0951-Techapp1.pdf).

We applied an indirect APPV $E^{\text {rns }}$ antibody ELISA, as described (9), and classified the serologic status into low $(\mathrm{S} / \mathrm{p} \leq 0.5)$, intermediate $(0.5<\mathrm{S} / \mathrm{p}<1.0)$, or highly ( $\mathrm{S} /$ $\mathrm{p} \geq 1.0$ ) reactive. Due to the lack of reference material and a standard assay, we could not determine test parameters (e.g., sensitivity, specificity) at this stage. Nevertheless, the ELISA was a valuable tool for detecting seroconversion in infected pigs; $\geq 60 \%$ of the animals showed intermediate to high reactivity in the antibody ELISA (Figure), which is in line with high APPV genome detection rates. We detected similar frequencies of APPV antibody-positive samples for each region, independent of the genome detection rates (Figure). We found most of the viral genomes $(\approx 86 \%)$ in samples with intermediate or low antibody status; few $(\approx 14 \%)$ of the highly antibodypositive animals were viremic at the same time. This 
observation might indicate a degree of protection provided by the induced antibodies. Of the $40 \%$ of the pigs that were antibody negative, $10 \%$ were genome positive; possible explanations are that serum samples were taken either from acutely infected animals before induction of a detectable antibody response or from persistently infected animals lacking a specific humoral immune response due to a specifically acquired immunotolerance, a well-known consequence of intrauterine pestivirus infections.

Our findings indicate that the recently discovered APPV is abundant on several continents. APPV must be regarded as a pig pathogen of likely worldwide relevance.

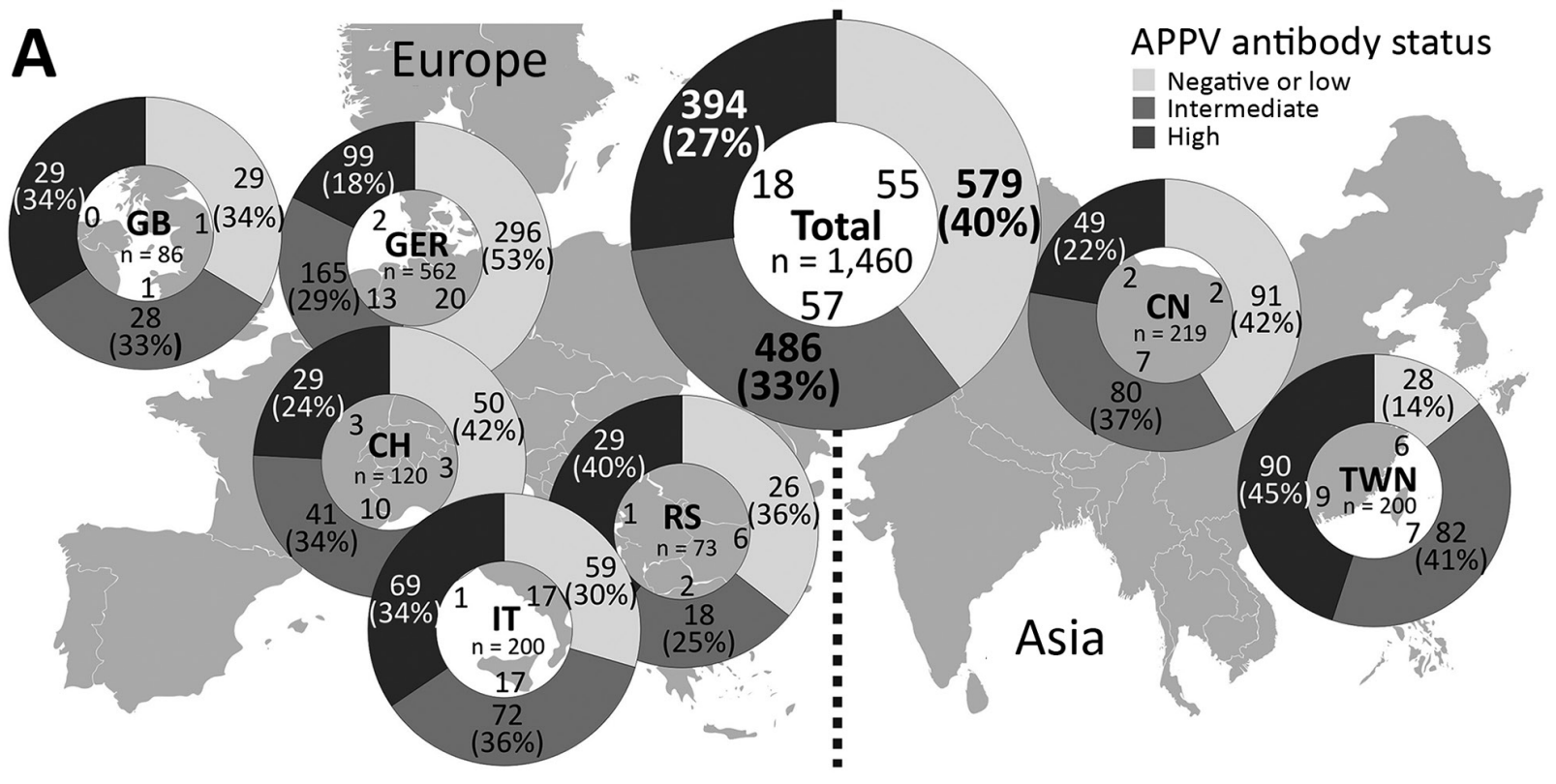

B

Switzerland_062_2015

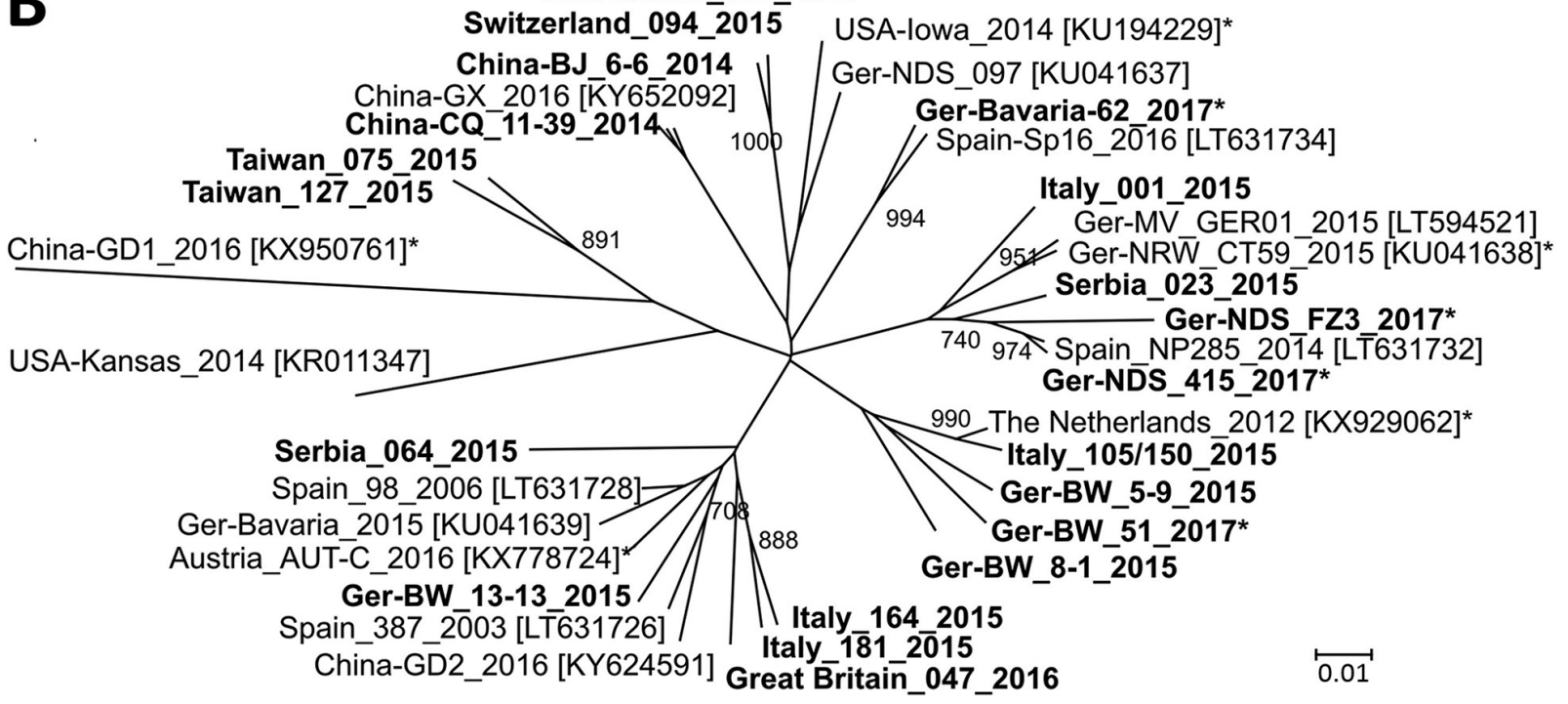

Figure. Detection rates of APPV genome and antibodies and genetic variability in Europe and Asia. A) APPV antibody status in pigs from parts of Europe and Asia. The region of origin, the number of investigated samples, and the absolute numbers of APPV genomepositive samples in dependence on the serologic category (low, intermediate, or high APPV antibody status) are shown in the central circle. B) Phylogenetic tree based on a 400-nt fragment in the nonstructural protein 3 encoding region. We calculated genetic distances using the Kimura 2-parameter model. We performed phylogenetic analysis by the neighbor-joining method including 1,000 iterations for bootstrap analysis. Only bootstrap values $\geq 700$ are indicated. Bold indicates sequences generated in this study; asterisks indicate sequences from piglets with congenital tremor. Accession numbers for reference sequences from GenBank are shown in brackets. Scale bar indicates nucleotide substitutions per site. APPV, atypical porcine pestivirus; $\mathrm{CH}$, Switzerland; CN, China; GB, Great Britain; GER, Germany; IT, Italy; RS, Serbia; TWN, Taiwan. 


\section{Acknowledgments}

We thank Tanja Frey and Birgitta Polley; the serum bank of the Federal Food Safety and Veterinary Office, Bern, Switzerland; the Animal and Plant Health Agency, Weybridge, UK; and the animal health service, Baden-Württemberg, Germany, for providing serum samples. We thank Daniela Indenbirken and Malik Alawi for their support in library preparation and analysis of next-generation sequencing data, and Elena Gräf for her support in Sanger sequencing. This work in part was financially supported by Boehringer Ingelheim.

Dr. Postel is a veterinarian and the head of the Laboratory for Molecular Biology of the European Union and World Organisation for Animal Health Reference Laboratory for Classical Swine Fever at the Institute of Virology of the University of Veterinary Medicine in Hannover, Germany. Research interests are molecular evolution and pathogenesis of pestiviruses and related viruses, characterization of novel pestivirus isolates, and diagnosis and control of classical swine fever.

\section{References}

1. Arruda BL, Arruda PH, Magstadt DR, Schwartz KJ, Dohlman T, Schleining JA, et al. Identification of a divergent lineage porcine pestivirus in nursing piglets with congenital tremors and reproduction of disease following experimental inoculation. PLoS One. 2016;11:e0150104. http://dx.doi.org/10.1371/ journal.pone. 0150104

2. de Groof A, Deijs M, Guelen L, van Grinsven L, van Os-Galdos L, Vogels W, et al. Atypical porcine pestivirus: a possible cause of congenital tremor type A-II in newborn piglets. Viruses. 2016; 8:271. http://dx.doi.org/10.3390/v8100271

3. Postel A, Hansmann F, Baechlein C, Fischer N, Alawi M, Grundhoff A, et al. Presence of atypical porcine pestivirus (APPV) genomes in newborn piglets correlates with congenital tremor. Sci Rep. 2016;6:27735. http://dx.doi.org/10.1038/srep27735

4. Schwarz L, Riedel C, Högler S, Sinn LJ, Voglmayr T, Wöchtl B, et al. Congenital infection with atypical porcine pestivirus (APPV) is associated with disease and viral persistence. Vet Res (Faisalabad). 2017;48:1. http://dx.doi.org/10.1186/ s13567-016-0406-1

5. British Veterinary Association. Congenital tremor associated with atypical porcine pestivirus. Vet Rec. 2017;180:42-3. http://dx.doi.org/10.1136/vr.j121

6. Hause BM, Collin EA, Peddireddi L, Yuan F, Chen Z, Hesse RA, et al. Discovery of a novel putative atypical porcine pestivirus in pigs in the USA. J Gen Virol. 2015;96:2994-8. http://dx.doi.org/ 10.1099/jgv.0.000251

7. Yuan J, Han Z, Li J, Huang Y, Yang J, Ding H, et al. Atypical porcine pestivirus as a novel type of pestivirus in pigs in China. Front Microbiol. 2017;8:862. http://dx.doi.org/10.3389/ fmicb.2017.00862

8. Beer M, Wernike K, Dräger C, Höper D, Pohlmann A, Bergermann C, et al. High prevalence of highly variable atypical porcine pestiviruses found in Germany. Transbound Emerg Dis. 2016. http://dx.doi.org/10.1111/tbed.12532

9. Postel A, Meyer D, Petrov A, Becher P. Recent emergence of a novel porcine pestivirus: interference with classical swine fever diagnosis? Emerg Microbes Infect. 2017;6:e19. http://dx.doi.org/ 10.1038/emi.2017.5

10. Isken O, Langerwisch U, Schönherr R, Lamp B, Schröder K, Duden $\mathrm{R}$, et al. Functional characterization of bovine viral diarrhea

virus nonstructural protein $5 \mathrm{~A}$ by reverse genetic analysis and live cell imaging. J Virol. 2014;88:82-98. http://dx.doi.org/10.1128/ JVI.01957-13

Address for correspondence: Paul Becher, EU and OIE Reference Laboratory for Classical Swine Fever, Institute for Virology, University of Veterinary Medicine Hannover, Buenteweg 17, 30559 Hannover, Germany; email: paul.becher@tiho-hannover.de

\section{Human Case of Streptococcus suis Disease, Ontario, Canada}

\section{Jeisa Gomez-Torres, Asim Nimir, James Cluett, Anita Aggarwal, Sameer Elsayed, Deirdre Soares, Sarah Teatero, Yan Chen, Marcelo Gottschalk, Nahuel Fittipaldi}

\begin{abstract}
Author affiliations: Tillsonburg District Memorial Hospital, Tillsonburg, Ontario, Canada (J. Gomez-Torres, A. Nimir); Tillsonburg Family Physicians, Tillsonburg (J. Cluett); Woodstock General Hospital, Woodstock, Ontario, Canada (A. Aggarwal, S. Elsayed); Western University, London, Ontario, Canada (S. Elsayed); Public Health Ontario Laboratory, Toronto, Ontario, Canada (D. Soares, S. Teatero, Y. Chen, N. Fittipaldi); University of Montreal, St-Hyacinthe, Quebec, Canada (M. Gottschalk); University of Toronto, Toronto (N. Fittipaldi)
\end{abstract}

DOI: https://doi.org/10.3201/eid2312.171005

We report a case of Streptococcus suis human disease in Ontario, Canada, caused by a serotype 2 strain genotypically similar to those commonly isolated from pigs in North America. Initially, the isolate was misidentified as a viridans group Streptococcus. Human S. suis infections may be underdiagnosed in North America.

Ctreptococcus suis is a zoonotic agent responsible for $\mathcal{O}_{\text {both sporadic and outbreak human disease in several }}$ Asian countries (1-3). However, human S. suis infections are less frequent in Western countries, and particularly in North America $(3,4)$. We describe a severe human S. suis infection in Ontario, Canada.

The patient, a 69-year-old male farmer, was brought to the emergency department of a rural community hospital in southwestern Ontario after being found unresponsive by his 